Proc Indian Natn Sci Acad $\mathbf{8 1}$ No. 1 February 2015 Special Issue, pp. 22-27

(C) Printed in India.

DOI:10.16943/ptinsa/2015/v81i1/48046

\title{
Nuclear Suppression of Muons at Forward Rapidity at Relativistic Heavy Ion Collisions UMME JAMIL $^{1, *}$, RAKTIM ABIR ${ }^{2}$, SOMNATH DE $^{3}$, MUNSHI G MUSTAFA $^{4}$ and DINESH K SRIVASTAVA ${ }^{3}$ \\ ${ }^{1}$ Debraj Roy College, Golaghat, Assam 785 621, India \\ ${ }^{2}$ Wayne State University, Detroit, Michigan, USA \\ ${ }^{3}$ Variable Energy Cyclotron Centre, Kolkata 700 064, India \\ ${ }^{4}$ Saha Institute of Nuclear Physics, Kolkata 700 064, India
}

(Received on 22 June 2014; Accepted on 2 September 2014)

\begin{abstract}
Heavy quarks produced in the initial stage of heavy ion collisions would traverse the quark gluon plasma, colliding with quarks and gluons and radiating gluons. In the process of collision with the quarks and gluons and also by radiation of gluons they lose energy. After their production, they may get fragmented into heavy mesons by picking up light quarks/antiquarks and in turn may decay through leptonic channels. These leptons would carry information of the initial stage of heavy ion collisions and also the evolution of the plasma.

In this work, we have made a detailed study for the nuclear modification factor of production of muons from the initial fusion of partons in a nuclear collision at LHC energy. We also present our result for the azimuthal anisotropy of heavy quark decay muons at LHC energy. We consider both the radiative and collision energy loss along with longitudinal expansion of the plasma for these calculations. We compare our results of muon $R_{A A}$ for $\mathrm{Pb}+\mathrm{Pb}$ collisions at 2.76 ATeV with ALICE data and results of muon $v_{2}$ for $\mathrm{Pb}+\mathrm{Pb}$ collisions at $2.76 \mathrm{ATeV}$ with ALICE preliminary data.
\end{abstract}

\section{Key Words : Heavy Ion Collisions; Heavy Quark; Energy Loss; Forward Rapidity; Nuclear Mod- ification; Azimuthal Anisotropy}

\section{Introduction}

Heavy quarks are produced from the fusion of gluons $(g g \rightarrow Q \bar{Q})$ or light quarks $(q \bar{q} \rightarrow Q \bar{Q})$, mainly during the initial stage of heavy ion collisions. Because of their large mass, their production at later times

\footnotetext{
*Author for Correspondence : E-mail: ummejamil@gmail.com
} 
is negligible. They also traverse the QGP without deviating much from the initial direction of production. So, they stands out in the sea of light quarks and gluons, which makes them an excellent probe for QGP. After production, they would traverse the QGP, colliding with quarks and gluons and radiating gluons before appearing as D or B mesons. These mesons further decay through leptonic channel. Thus the final spectra for leptons would contain information about the energy loss suffered by the heavy quarks. In a noncentral collision the heavy quarks produced from the initial fusion, moving out of the reaction plane, would cover different distances inside the plasma. Therefore, they would lose different amounts of energy and be quenched to a different extent. This will lead to an azimuthal anisotropy of momentum distribution of leptons.

In this work we calculate the $p_{T}$ distribution of heavy quarks from initial fusion and then calculate the final $p_{T}$ distribution taking into account the energy loss suffered by them as they pass through the QGP. Finally we perform a Monte Carlo calculation to obtain the average change in the transverse momentum spectra of heavy quarks for nucleus-nucleus collisions and get $R_{A A}$ as a function of $p_{T}$ for different rapidities. The azimuthal anisotropy of heavy flavour decay muons is calculated in $\mathrm{Pb}+\mathrm{Pb}$ collision at $2.76 \mathrm{ATeV}$ at $20-40 \%$ centrality in the rapidity window 2.5 to 4 .

\section{Initial Conditions and Energy Loss Formalisms}

The $p_{T}$ distribution of heavy quarks produced from the initial fusion of partons in nucleus-nucleus collisions at LHC energy is obtained by Fixed Order Next to Leading Logarithm (FONLL) calculation (Cacciari et al., 1998; Cacciari et al., 2001). CTEQ 6.6 structure function set for nucleons is used throughout the calculation. We take Peterson fragmentation function (Peterson et al., 1983) with parameter $\epsilon_{c}=0.06$ and $\epsilon_{b}=0.006$ for fragmentation of $\mathrm{c}$ quarks into D-mesons and b quarks into B-mesons, respectively. The central particle rapidity density is taken as $\approx 2850$ for $\mathrm{Pb}+\mathrm{Pb}$ collisions at $2.76 \mathrm{ATeV}$ (Aamodt et al., 2010).

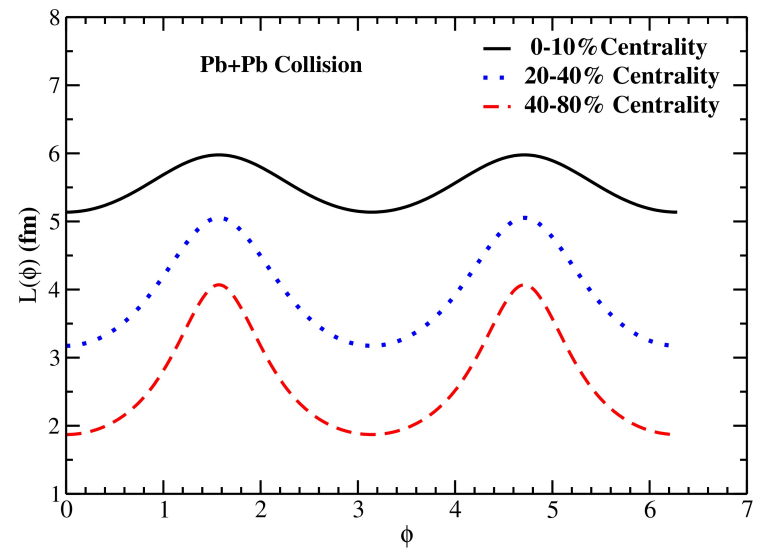

Fig. 1: Variation of average path length $L(\phi)$ with azimuthal angle $\phi$ 
In non-central collisions, the azimuthal dependence of the average path length for different impact parameter is to be considered. We consider a heavy quark after production at the point $(x, y)$, moves at an angle $\phi$ with respect to the reaction plane in a non-central collision with impact parameter b. It traverses the distance $1(\mathrm{x}, \mathrm{y}, \phi, \mathrm{b})$ inside the plasma. Using a simple approach, based on Glauber model, we evaluate the dependence of the average path-length on the azimuthal angle with respect to the reaction plane. Assuming uniform densities for the colliding nuclei, the average path-length for an impact parameter $\mathrm{b}$ and azimuthal angle $\phi$ can be written as (De and Srivastava, 2012):

$$
\langle L(\phi ; b)\rangle=\frac{\iint l(x, y, \phi, b) T_{A B}(x, y ; b) \mathrm{dx} \mathrm{dy}}{\iint T_{A B}(x, y ; b) \mathrm{dx} \mathrm{dy}} .
$$

Where $T_{A B}(x, y ; b)=T_{A}(x+b / 2, y) T_{A}(x-b / 2, y)$ is the nuclear overlap function and $t_{A}$ and $t_{B}$ are the transverse density profiles of the two nuclei. An average of $L(\phi ; b)$ over $\phi$ (varying from zero to $2 \pi$ ) gives the average path length $\mathrm{L}(\phi)$ (Fig. 1). As the heavy quarks lose most of their energy in interaction with gluons, it is enough to consider only the distribution of gluons. Their density at the time $\tau$ is given by $\rho(\tau)$ and the corresponding temperature, assuming a chemically equilibrated plasma is given by $T(\tau)$ (Djordjevic and Gyulassy, 2004; Wicks et al., 2007):

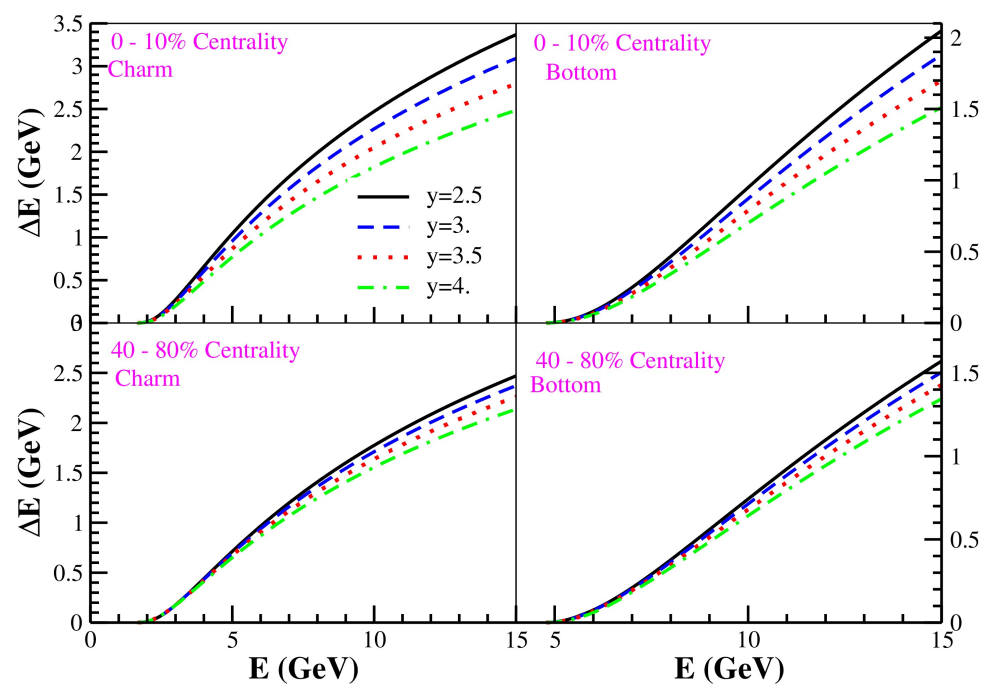

Fig. 2: Radiative energy loss suffered by charm and bottom quarks while passing through the QGP at forward rapidities at different centralities

$$
\rho(\tau)=\frac{1}{\pi R^{2} \tau} \frac{d N_{g}}{d y}, \quad T(\tau)=\left(\frac{\pi^{2}}{1.202} \frac{\rho(\tau)}{\left(9 N_{f}+16\right)}\right)^{\frac{1}{3}}
$$


The initial time of formation of QGP, $\tau_{0}$ is taken as $0.2 \mathrm{fm} / \mathrm{c}$. We approximate the expanding and cooling plasma with one at a temperature of $\mathrm{T}(\tau)$ at $\tau=\langle\mathrm{L}\rangle_{\mathrm{eff}} / 2$, where $\langle\mathrm{L}\rangle_{\mathrm{eff}}=\min \left[\langle\mathrm{L}\rangle, v_{T} \times \tau_{c}\right]$, where $v_{T}$ is the transverse velocity of the heavy quark and $\tau_{c}$ is the critical temperature (Jamil and Srivastava, 2010). We consider the formalism developed by Peigne and Peshier (PP) (Peigne and Peshier, 2008) to calculate the collisional energy loss. The radiative energy loss is calculated using a recent formalism, which we will refer as 'Present', (Abir et al., 2012) and also by the formalism developed by Djordjevic, Gyulassy, Levai and Vitev (DGLV) (Djordjevic and Gyulassy, 2004; Wicks et al., 2007).

In Fig. 2, we plot the radiative energy loss of charm and bottom quarks at $0-10 \%$ and $40-80 \%$ centralities by Present formalism as a function of transverse energy at $2.76 \mathrm{ATeV}$ at LHC. Each of the plots of Fig. 2 is presented for different rapidities in the rapidity window 2.5 to 4 .



A

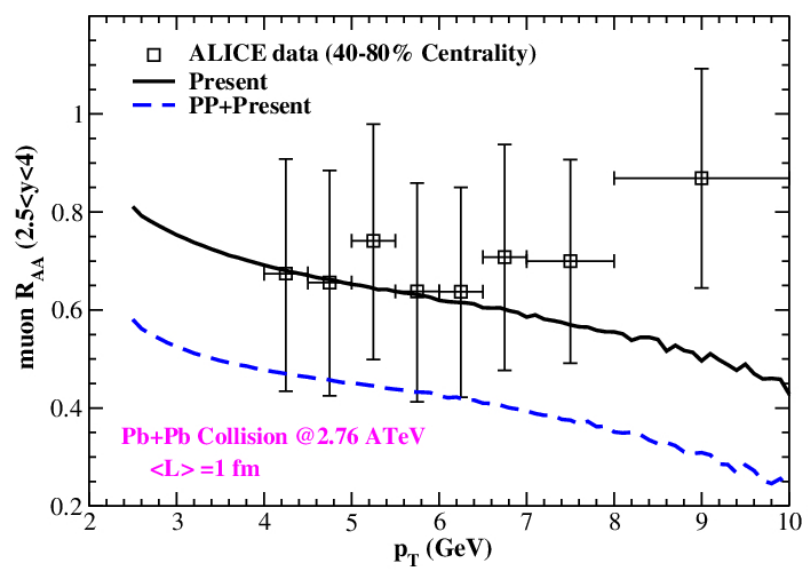

B

Fig. 3: (A) $R_{A A}$ of muons at forward rapidity at $0-10 \%$ centrality. (B) Same as left panel at $40-80 \%$ centrality

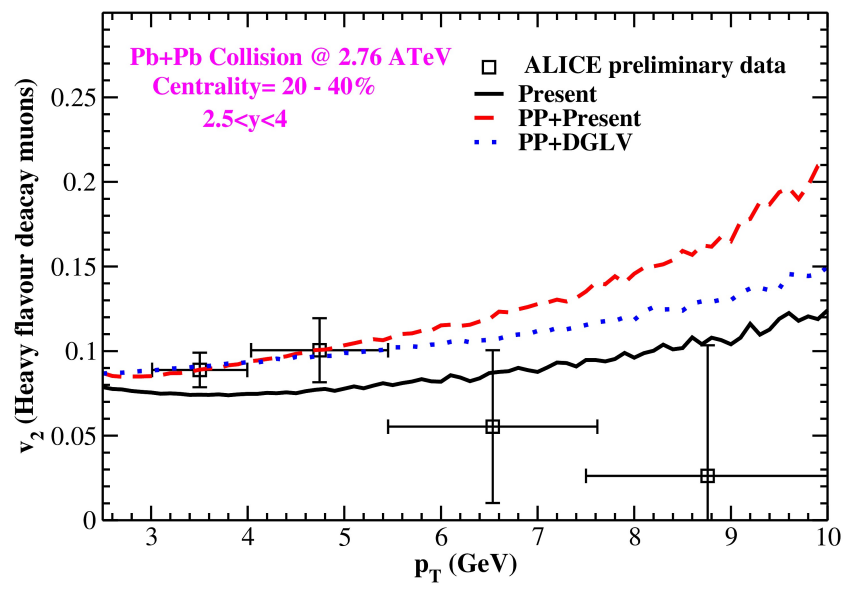

Fig. 4: $v_{2}$ of muons at $2.76 \mathrm{ATeV}$ for $20-40 \%$ centrality 


\section{$R_{A A}$ of Muons}

The results of nuclear modification factor of muons at different centrality with both collisional and radiative energy loss at $2.76 \mathrm{ATeV}$ at LHC are presented in Fig. 3. Our result at 0-10 centrality with only Present formalism found to agree well with the recent data (Abelev et al., 2012) from ALICE collaboration. Whereas our result with Present formalism at $40-80 \%$ centrality predicts more suppression for $p_{T}>8 \mathrm{GeV}$. We also observe that the inclusion of the collisional energy loss further suppresses the nuclear modification factor significantly in both the cases and predicts more suppression compared to the experimental result. Comparison of the predictions of $R_{A A}$ by DGLV and Present formalisms can be found in reference (Abir $e t$ al., 2012) with detail discussion.

\section{Azimuthal Anisotropy}

The differential azimuthal anisotropy is measured in terms of the parameter $v_{2}\left(p_{T}\right)$, given by:

$$
v_{2}\left(p_{T}\right)=\frac{\int_{0}^{2 \pi} \mathrm{d} \phi \cos (2 \phi) d N / d^{2} p_{T} d y}{\int_{0}^{2 \pi} \mathrm{d} \phi d N / d^{2} p_{T} d y} .
$$

The result of azimuthal anisotropy of muons at $2.76 \mathrm{ATeV}$ for $20-40 \%$ centrality is presented in Fig. 4 . We compare the predicted result with the preliminary ALICE data of heavy flavour decay muons $v_{2}\left(p_{T}\right)$ (Zhang. 2013). We need more experimental data for $v_{2}\left(p_{T}\right)$ at high $p_{T}$ to understand our predicted trend.

\section{Summary}

We found that our prediction for $R_{A A}$ considering only radiative energy loss agrees well with the ALICE data. But when we consider both the radiative and collisional energy losses, our result predicts more suppression than expected. This is due to the consideration of constant density distribution of the colliding nuclei while calculating the average path length traversed by the quark. This means that we are predicting the maximum possible energy loss as well as suppression. We expect that the consideration of Wood-saxon density distribution for the colliding nuclei will improve the scenario.

\section{References}

1. Aamodt K et al., (ALICE Collaboration) (2010) Charged-Particle Multiplicity Density at Midrapidity in Central $\mathrm{Pb}-\mathrm{Pb}$ Collisions at ?sNN $=2.76 \mathrm{TeV}$ in Physical Review Letters 105252301 
2. Abelev B et al., (ALICE Collaboration) (2012) Production of Muons from Heavy Flavor Decays at Forward Rapidity in pp and $\mathrm{Pb}-\mathrm{Pb}$ Collisions at $\sqrt{s_{N N}}=2.76 \mathrm{TeV}$ in Physical Review Letters 109112301

3. Abir Raktim, Jamil Umme, Mustafa Munshi G, Srivastava Dinesh K (2012) Heavy quark energy loss and D-mesons in RHIC and LHC energies in Physics Letters B 715 183-189

4. Cacciari M, Greco M and Nason P (1998) The pT Spectrum in Heavy Flavor Hadroproduction in Journal of High Energy Physics $\mathbf{9 8 0 5} 007$

5. Cacciari M, Frixione S and Nason P (2001) The pT Spectrum in Heavy Flavor Photoproduction in Journal of High Energy Physics 0103006

6. De Somnath and Srivastava Dinesh K (2012) System size dependence of nuclear modification and azimuthal anisotropy of jet quenching in Journal of Physics $G \mathbf{3 9} 015001$

7. Djordjevic M and Gyulassy M (2004) Heavy quark radiative energy loss in QCD matter in Nuclear Physics A $733265-298$

8. Jamil Umme and Srivastava Dinesh K (2010) Nuclear suppression of heavy-quark production at forward rapidities in relativistic heavy-ion collisions in Journal of Physics G 37085106

9. Peign S, Peshier A (2008) Collisional energy loss of a fast heavy quark in a quark-gluon plasma in Physical Review D 77114017

10. Peterson C, Schlatter D, Schmitt I and Zerwas PM (1983) Scaling violations in inclusive e+e? annihilation spectra in Physical Review D 27105

11. Wicks S, Horowitz W, Djordjevic M and Gyulassy M (2007) Elastic, inelastic, and path length fluctuations in jet tomography in Nuclear Physics A $\mathbf{7 8 4}$ 426-442

12. Zhang Xiaoming for the ALICE Collaboration (2013) Nuclear Modification Factor and Elliptic Flow of Muons from Heavy-flavour Decays in $\mathrm{Pb}-\mathrm{Pb}$ Collisions at $\sqrt{s_{N N}}=2.76 \mathrm{TeV}$ with ALICE, presented in Strangeness in Quark Matter, Bermingham, U.K. 